University of Nebraska - Lincoln

DigitalCommons@University of Nebraska - Lincoln

2000

\title{
The Polarization of Sb Overlayers on $\mathrm{NiMnSb}(100)$
}

Takashi Komesu

University of Nebraska-Lincoln, tkomesu2@unl.edu

C.N. Borca

University of Nebraska-Lincoln

Hae-Kyung Jeong

University of Nebraska-Lincoln, hjeong@unl.edu

Peter A. Dowben

University of Nebraska-Lincoln, pdowben@unl.edu

Delia Ristoiu

CNRS Laboratoire Louis Nee'l, 25 aĺenue des Martyrs BP 166, 38042 Grenoble CEDEX 09, France

See next page for additional authors

Follow this and additional works at: https://digitalcommons.unl.edu/physicsdowben

Part of the Physics Commons

Komesu, Takashi; Borca, C.N.; Jeong, Hae-Kyung; Dowben, Peter A.; Ristoiu, Delia; Nozieres, J.P.; Stadler, Shane; and Idzerda, Y.U., "The Polarization of Sb Overlayers on NiMnSb(100)" (2000). Peter Dowben Publications. 225.

https://digitalcommons.unl.edu/physicsdowben/225

This Article is brought to you for free and open access by the Research Papers in Physics and Astronomy at DigitalCommons@University of Nebraska - Lincoln. It has been accepted for inclusion in Peter Dowben Publications by an authorized administrator of DigitalCommons@University of Nebraska - Lincoln. 


\section{Authors}

Takashi Komesu, C.N. Borca, Hae-Kyung Jeong, Peter A. Dowben, Delia Ristoiu, J.P. Nozieres, Shane Stadler, and Y.U. Idzerda 


\title{
The polarization of $\mathrm{Sb}$ overlayers on $\mathrm{NiMnSb}(100)$
}

\author{
Takashi Komesu $^{\text {a }, \text { C.N. Borca }}{ }^{\mathrm{a}}$, Hae-Kyung Jeong ${ }^{\mathrm{a}}$, P.A. Dowben ${ }^{\mathrm{a}, *}$, Delia Ristoiu ${ }^{\mathrm{b}}$, \\ J.P. Nozières ${ }^{b}$, Shane Stadler ${ }^{c}$, Y.U. Idzerda ${ }^{c}$ \\ ${ }^{a}$ Department of Physics and Astronomy and the Center for Materials Research and Analysis, Behlen Laboratory of Physics, \\ University of Nebraska-Lincoln, Lincoln, NE 68588-0111, USA \\ ${ }^{\mathrm{b}}$ CNRS Laboratoire Louis Neél, 25 avenue des Martyrs BP 166, 38042 Grenoble CEDEX 09, France \\ ${ }^{\mathrm{c}}$ Naval Research Laboratory, Materials Physics Branch, Washington DC 20375, USA
}

Received 3 April 2000; accepted 13 July 2000

Communicated by L.J. Sham

\begin{abstract}
We have investigated the induced polarization of paramagnetic Sb overlayers on the Heusler alloy NiMnSb. From combined X-ray absorption spectroscopy (XAS) and spin-polarized inverse photoemission spectroscopy (SPIPES), we can assign some of the unoccupied states of the Heusler alloy NiMnSb. With increasing thickness of the Sb overlayer, there is a decline in the density of states near the Fermi energy, as expected for a semimetal overlayer on a metallic substrate. While the $\mathrm{Sb}$ is polarized by the ferromagnetic NiMnSb substrate, consistent with the expectations of mean field theory, the polarization at the center of the surface/overlayer Brillouin zone cannot be easily related to the induced magnetization. (C) 2000 Elsevier Science B.V. All rights reserved.
\end{abstract}

PACS: 71.20.Lp; 73.20.At; 75.25. + z

Keywords: NiMnSb Heusler alloys; Inverse photoemission; Angle resolved XPS; Surfaces

It is generally expected from Landau-Ginzburg mean field theory [1-3], that a ferromagnetic substrate will induce a magnetic moment in a paramagnetic metallic overlayer leading to a net polarization of that overlayer. This induced magnetization, to a very good approximation should vary as

$M(z)=M(0) \exp (-\kappa z)$

where $z$ is the overlayer thickness, and $M(z)$ is a magnetization of surface or surface region, and $\kappa$ is

\footnotetext{
* Corresponding author. Tel.: +1 402472 2770; fax: +1 402 4722879.

E-mail address: pdowben@unl.edu (P.A. Dowben).
}

a reciprocal of the paramagnetic correlation length of the overlayer. Thus, the surface magnetization decays exponentially as overlayer thickness increases.

While polarization at the Brillouin zone center $\left(\boldsymbol{k}_{\|}=0\right)$ does corresponds to long range magnetic order, it is not clear that polarization is, in fact, easily related to the magnetic moment for any given system. A extreme test of this issue is to study a paramagnetic overlayer with a low density of state at the Fermi energy (a semimetal or semiconductor like antimony $[4,5]$ ) on a substrate with a very high level of polarization at the Fermi level (ideally a material such as a half metallic system: metallic in spin- 
majority and insulating in spin-minority). The applicability of Landau-Ginzburg models require that the induced magnetization in the overlayer be small and that there is only weak enhancement of the magnetization at the overlayer free surface. Antimony (Sb) fulfills these conditions.

While it has been suggested that NiMnSb is a half metallic system [6-8], it is not clear that this is the case at finite temperatures [9-15]. Nonetheless, it is clear that with the appropriate surface preparation, the polarization of $\mathrm{NiMnSb}(100)$, at the Brillouin zone center $\left(\boldsymbol{k}_{\|}=0\right)$, is very high and close to $100 \%$ at finite temperature [9]. Furthermore, the $\mathrm{Sb}$, in the $\mathrm{NiMnSb}$ has little, if any, moment judging from the MCD signal (Fig. 3c), neutron diffraction [16] and theory [8], so that an induced moment from an $\mathrm{Sb}$ overlayer can easily be identified. Amongst the other potential half-metallic systems, the manganese perovskites have complex surface structures [17-21] and $\mathrm{CrO}_{2}$ surface is poorly characterized [22], so are less suitable, at present, for such studies.

Epitaxial (110)MgO/(100)Mo/(100)NiMnSb thin films were grown by facing targets sputtering. Details of the growth conditions and film structure can be found elsewhere [23]. A $1000 \AA$ Sb capping layer was added to prevent oxidation of the $\mathrm{NiMnSb}$ crystalline films and provide the basis for the paramagnetic film overlayer. Starting from a fully capped layer is important because the surface of $\mathrm{NiMnSb}(100)$ is very fragile [9] and preparation of the stoichiometric surface followed by deposition of the paramagnetic overlayer is more likely to lead to a complicated interface.

After the sample surfaces were cleaned in ultra high vacuum by repeated $\mathrm{Ar}^{+}$sputtering (pressure of $1 \times 10^{-6}$ Torr) and annealing to $450 \mathrm{~K}$ the surface was found to be free of both oxygen and carbon. With $\mathrm{Ar}^{+}$sputtering (to remove surface contamination) followed by a flash anneal to $700 \mathrm{~K}$ (to remove the excess $\mathrm{Sb}$ ), the stoichiometric surface can be prepared [9] and the LEED pattern is consistent with the $5.9 \AA$ (here $6.0 \pm 0.1 \AA$ ) lattice constant of NiMnSb.

The $\mathrm{Sb}$ capping layers grows epitaxially on $\mathrm{NiMnSb}$ with a $\langle 100\rangle$ orientation, a cubic Pm3m structure and a $3.1 \AA$ lattice constant. The crystallinity and orientation of the NiMnSb were established $e x$-situ by X-ray diffraction [23] and, once the capping layers were removed, again by low energy electron diffraction (LEED) [9].

The spin-polarized inverse photoemission experiments were undertaken with a transversely polarized spin electron gun based upon the Ciccacci design [24] as described elsewhere [25,26]. The spin electron gun was designed in a compact form on a separate chamber equipped with an iodine based Geiger-Müller isochromat photon detector with an $\mathrm{SrF}_{2}$ window. As is typical of such instruments, the electron gun has $28 \%$ spin-polarization, and the data has been corrected for this incident gun polarization. The direction of electron polarization is in the plane of the sample for all incidence angles, as is the applied field, and spectra were obtained at remanence. The energy resolution was in the vicinity of $400 \mathrm{meV}$ and the wave-vector uncertainty is \pm 0.025 $\AA^{-1}$ for these measurements. The field was applied along the in-plane $\langle 100\rangle$ of $\mathrm{NiMnSb}(100)$ with magnitudes in excess of $400 \mathrm{Oe}$, far larger than the samples' saturation and coercive fields of about 40 and 32 Oe respectively (characterized by magnetooptic Kerr effect, ex situ [9]). The Fermi level was established from tantalum foils in electrical contact with the sample. The conduction band features are reported with respect to this Fermi level and emission angle (or incidence angle in the case of the inverse photoemission) is with respect to the surface normal. Typically, several experiments are summed, to improve the signal to noise ratio in the spinpolarized inverse photoemission spectra.

Angle resolved X-ray photoemission spectroscopy (ARXPS) of the $\mathrm{Sb}, \mathrm{Mn}$ and $\mathrm{Ni}$ core levels was undertaken with the $\mathrm{Mg}-\mathrm{K}_{a}$ line $(1253.6 \mathrm{eV})$ on a number of different samples. Energy distribution curves of the elemental Ni $\left(2 \mathrm{p}_{3 / 2}\right), \mathrm{Mn}\left(2 \mathrm{p}_{3 / 2}\right.$ and $\left.2 \mathrm{p}_{1 / 2}\right)$, and $\mathrm{Sb}\left(3 \mathrm{~d}_{5 / 2}\right.$ and $\left.3 \mathrm{~d}_{3 / 2}\right)$ core levels were acquired with a large hemispherical electron energy analyzer and the intensities were measured, as were the binding energies, as a function of emission angle, with respect to the surface normal. Intensities are normalized by the cross-section, as calculated by Scofield for an excitation energy of $1253.6 \mathrm{eV}(\mathrm{Mg}-$ $\mathrm{K}_{a}$ ) [27] and by the transmission function of the electron analyzer [28], as noted in more extensive detail elsewhere [9,29].

The X-ray adsorption (XAS) and magnetic circular dichroism (MCD) spectra were recorded by moni- 
toring the sample current, which corresponds to measuring the total electron yield, under a 400 Oe pulsed magnetic field applied along the in-plane (100) axis. The spectra for two opposite helicities were recorded by alternating the magnetization at every photon energy. In total electron yield mode, the XAS spectra probes approximately 20 to $200 \AA$ in depth, which is an intermediate length scale between the bulk and the surface boundary. The samples used in the XAS measurements were capped with $25 \AA$ of $\mathrm{Al}$ (not $\mathrm{Sb}$ ) so as to only probe the NiMnSb substrate, without contributions from the overlayer cap. The conduction band edge (the approximate Fermi energy), for the XAS spectra is assigned on the basis of the experi- mentally measured core level binding energies for $\mathrm{NiMnSb}(100)$ : $528.2 \mathrm{eV}$ for the $\mathrm{Sb} 3 \mathrm{~d}_{5 / 2}, 640.2 \mathrm{eV}$ for the Mn $2 \mathrm{p}_{3 / 2}$ and $853.3 \mathrm{eV}$ for the $\mathrm{Ni} 2 \mathrm{p}_{3 / 2}$.

As increasing amounts of the $\mathrm{Sb}$ overlayer are removed, the Mn and Ni XPS signals increase (Fig. 1). The maximum intensities for the $\mathrm{Mn}\left(2 \mathrm{p}_{3 / 2}+\right.$ $\left.2 \mathrm{p}_{1 / 2}\right)$ and $\mathrm{Ni}\left(2 \mathrm{p}_{3 / 2}\right)$ core levels are obtained for a clean surface, when attenuation by the $\mathrm{Sb}$ overlayer is minimized.

We have found that a flash anneal to $700 \mathrm{~K}$ removes the excess $\mathrm{Sb}[9,22]$. The resulting surface is relatively unreactive (a low sticking coefficient for contaminants) and exhibits a sharp LEED pattern, with a $6.0 \pm 0.1 \AA$ surface lattice constant. Fig. 1
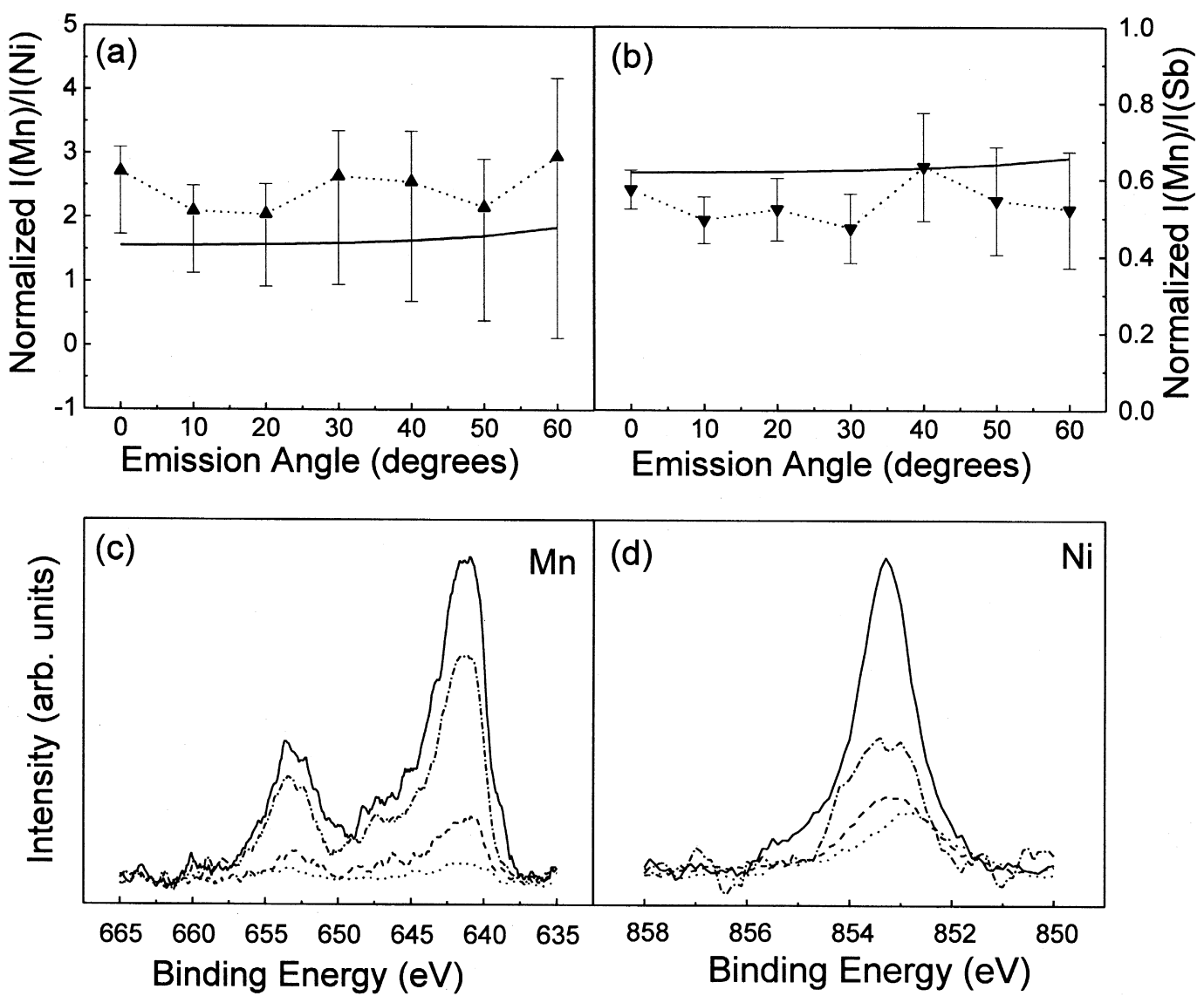

Fig. 1. Angle resolved X-ray photoemision intensity ratios of the normalized (a) (Mn $\left.2 \mathrm{p}_{3 / 2}+2 \mathrm{p}_{1 / 2}\right) /\left(\mathrm{Ni} 2 \mathrm{p}_{3 / 2}\right)$ and (b) $\left(\mathrm{Mn} 2 \mathrm{p}_{3 / 2}+\right.$ $\left.2 \mathrm{p}_{1 / 2}\right) /\left(\mathrm{Sb} \mathrm{3d}_{5 / 2}\right)$ for the clean stoichiometric surface of NiMnSb(100). The continuous lines represent model fits that assumes a Mn-Sb composition for the top surface layer of the alloy. The Sb thickness dependence of the (c) Mn $\left(2 \mathrm{p}_{3 / 2}+2 \mathrm{p}_{1 / 2}\right)$ and (d) Ni $\left(2 \mathrm{p}_{3 / 2}\right)$ core levels intensities, as also shown, for normal emission geometry. The highest intensity in both cases (continuous line) is obtained from the clean stoichiometric NiMnSb(100) surface. (The Mn and Ni peak intensities decrease as the thickness of the Sb capping layer increases). 
presents the peak ratios from angle resolved photoemission spectroscopy (ARXPS) for the normalized intensities of the (a) $\mathrm{I}(\mathrm{Mn}) / \mathrm{I}(\mathrm{Ni})$ and (b) $\mathrm{I}(\mathrm{Mn}) /$ $\mathrm{I}(\mathrm{Sb})$ following removal of all the excess $\mathrm{Sb}$. The data are compared to the calculated intensity ratios for the stoichiometric ordered $\mathrm{NiMnSb}(100)$ clean surface, with MnSb termination [9,22].

The unoccupied electronic structure of this clean $\mathrm{NiMnSb}(100)$ surface was probed with spin-polarized inverse photoemission spectroscopy (SPIPES) (Fig. 2a), and X-ray absorption spectroscopy (XAS) for Mn 2p (Fig. 2b) and for Ni 2p (Fig. 2c). Both MCD and XAS spectra are very sensitive to the unoccupied levels, and can be roughly compared to inverse

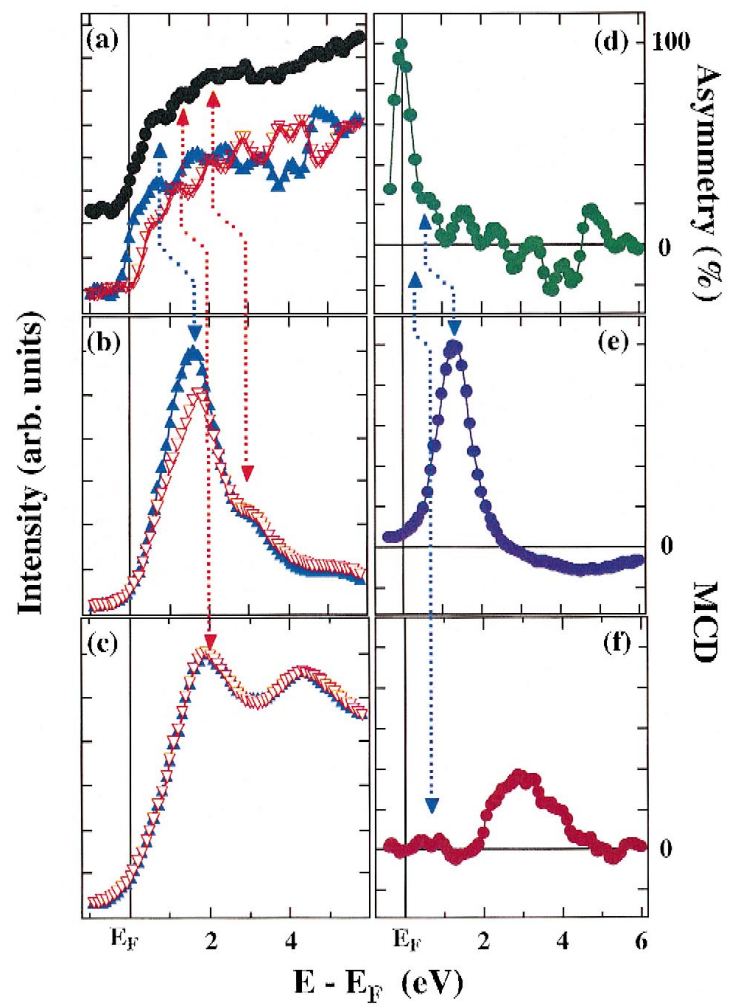

Fig. 2. The comparison of the SPIPES spectra, MCD and XAS spectra for $\mathrm{NiMnSb}(100)$. The spin-polarized inverse photoemission (SPIPES) $(\Delta \nabla)$ and integrated $(\bullet)$ for the stochiometric $\mathrm{NiMnSb}(100)$ clean surface at $300 \mathrm{~K}$ (a) results can be compared X-ray absorption results of Mn $2 p$ (b) and Ni $2 p$ (c) electrons. The graph (d) indicates the polarization asymmetry from SPIPES (d) is compared to the MCD data from across Mn 2p (e) and Ni 2p (d) respectively. The dashed lines indicate the possible corresponding unoccupied states orbitals in SPIPES with the XAS or MCD results.

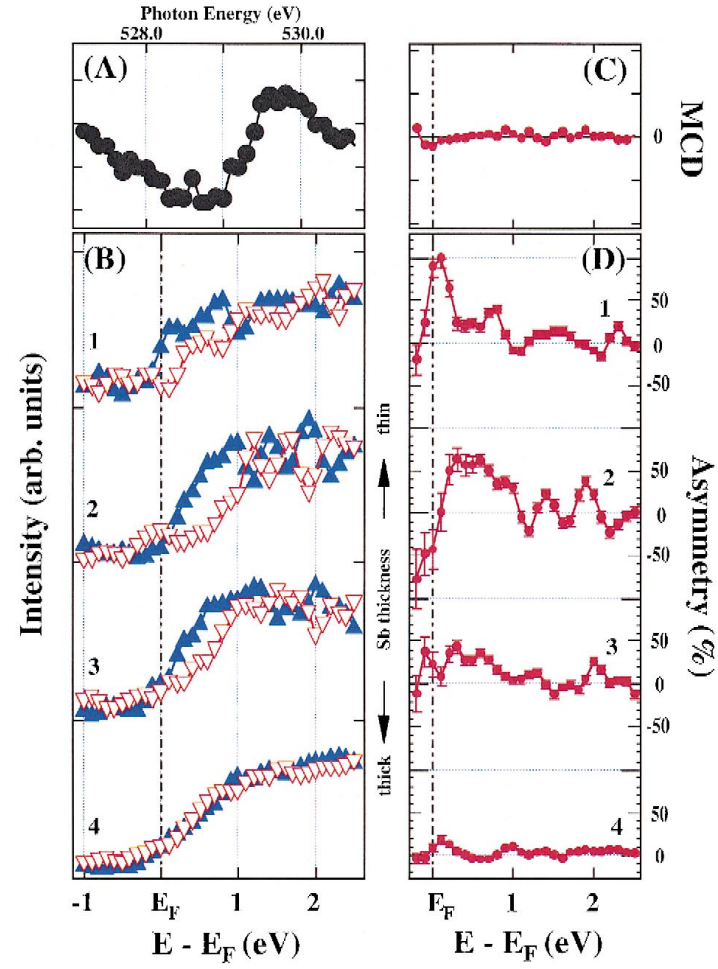

Fig. 3. Spin-polarized inverse photoemission spectra (SPIPES) and polarization asymmetries are shown for increasing $\mathrm{Sb}$ overlayer thickness (B2, B3 to B4 and D2, D3, to D4 respectively) where spectra $\mathrm{B} 1$ and polarization asymmetry $\mathrm{D} 1$ are for the stochiometric NiMnSb(100) surface. As the overlayer thickness increases, comparison of the SPIPES spectra can be increasingly compared to the X-ray absorption spectroscopy (XAS), taken at $300 \mathrm{~K}$, across $\mathrm{Sb} 3 \mathrm{~d}$ core (A), though contributions from the unoccupied $\mathrm{Sb} 6 \mathrm{~s}$ and $5 \mathrm{~d}$ rectangular representations are (because of selection rules) weak. The Sb MCD signal (C), across the $3 \mathrm{~d}$ core, indicates that the clean $\mathrm{NiMnSb}(100)$ substrate has little or no Sb magnetic moment. The spectra for different thickness of Sb (B2, B3 to B4) and corresponding polarization asymmetries (D2, D3, to D4 respectively)were acquired at roughly $200 \mathrm{~K}$, while the clean surface of stoichiometric $\mathrm{NiMnSb}(100)$ was undertaken at about 300 K.

photoemission, once the core level binding energy is taken into account.

In spin-polarized inverse photoemission, spinmajority states $(\boldsymbol{\Delta})$, i.e. parallel to applied magnetic field and spin-minority states $(\nabla)$, i.e. antiparallel to the applied magnetic field, are plotted, as well as the spin-integrated density of states $(\mathbf{)})$. The polarization of the $\mathrm{NiMnSb}(100)$ surface is determined by the difference of spin up and down in SPIPES 
(Fig. 2d). Subject the necessary selection rules, core excitation spectra reflect the joint density of states between the core level and the unoccupied states. These results are with the MCD signal (the difference of XAS signal with left and right circularly polarized light), as seen in Fig. 2e,f. Note that in the XAS spectra, the unoccupied states are shifted to slightly higher energies above $E_{\mathrm{F}}$ than in SPIPES, as expected from the perturbation of the Coulombic interaction with the photoexcitation core hole. Nonetheless, from this comparison, we can make a rough assignment of the unoccupied states in inverse photoemission, as has been undertaken with manganese perovskites [30]. The density of states just above $E_{\mathrm{F}}$ for $\mathrm{NiMnSb}(100)$ is largely $\mathrm{Mn}$ in origin and $\mathrm{Mn}$ has the largest polarization near $E_{\mathrm{F}}$ (nearly $100 \%$ above background for $\boldsymbol{k}_{\|}=0$ ), with only a small contribution from $\mathrm{Ni}$ (Fig. 2) and virtually none from $\mathrm{Sb}$ (Fig. 3). This is consistent with theory [6-8] and experiment [16].

Since dipole selections rules can be applied to the excitation determine the orbital symmetry (angular momentum) of the unoccupied orbitals, or intermediate (core exciton) excited state, and the typical one electron transition requires that $\Delta l= \pm 1$, and $\Delta m_{l}$ $= \pm 1,0$. For states above the Fermi level, the band assignments can be experimentally determined in polarization dependent XAS studies. The selection rules (applicable to both the $\mathrm{Ni}$ and $\mathrm{Mn}$ XAS and MCD spectra) from a core $p$ shell to the unoccupied orbitals, with circularly polarized light, are possible to states of $\mathrm{d}_{z^{2}}, \mathrm{~d}_{y^{2}}, \mathrm{~d}_{x^{2}}, \mathrm{~d}_{x z}, \mathrm{~d}_{y z}$, and $\mathrm{d}_{x y}$ character in $\mathrm{C}_{2 \mathrm{v}}$ (the local two fold symmetric point group for the surface, though consideration of the bulk point group symmetry should not be excluded for XAS). Unfortunately, we have currently insufficient light polarization dependent data for a more refined symmetry assignment of the unoccupied states. Inverse photoemission is restricted to the unoccupied final $\mathrm{d}_{x z}, \mathrm{~d}_{y z}$ and $\mathrm{d}_{z^{2}}$ states (as well as $\mathrm{s}, \mathrm{p}_{z}, \mathrm{p}_{x}$, and $\mathrm{p}_{y}$ ) because the initial state is the free electron $\left(a_{1}\right.$ or the fully symmetry initial state) at normal incidence (the highest symmetry point in $\mathrm{C}_{2 \mathrm{v}}$ ). For this reason, the major unoccupied states cannot be solely of $\mathrm{d}_{z^{2}}, \mathrm{~d}_{y^{2}}$, $\mathrm{d}_{x y}$ because of the agreement of the XAS features with the inverse data. Hybridization cannot be excluded (in XAS) with states of the other rectangular representations.
The thickness dependence of surface polarization of paramagnetic $\mathrm{Sb}$ on the top of ferromagnet $\mathrm{NiMnSb}(100)$ is shown in Fig. 3, based on SPIPES results. The thick antimony overlayer surface exhibits virtually no net polarization in spin-polarized inverse photoemission. As the Sb overlayer thickness is decreased (through sputtering), there is an increase in the polarization asymmetry, in spin-polarized inverse photoemission, particularly near the Fermi energy (Fig. 2). This polarization asymmetry is further enhanced by decreasing the sample temperature from $300 \mathrm{~K}$ to $200 \mathrm{~K}$, as noted elsewhere [9].

The polarization asymmetry can be caused by the NiMnSb substrate (if the electron have sufficient mean free path) or by induced polarization of the overlayer by the NiMnSb substrate. While inverse photoemission is extremely surface sensitive, the $\mathrm{NiMnSb}$ signal attenuation is a concern because with a lower density of states near the Fermi level (in Sb), there is a tendency for the electron mean free path to increase. Calculated models for the mean free path are typically based on the Bethe equation [31]. Thus at low kinetic energies, the mean free path should strongly depend upon the inverse of the free electron plasmon energy squared ( $\left.\alpha E_{\mathrm{p}}^{-2}\right)$ or, alternatively, roughly proportional to the inverse of the number of valence electrons [32-34]. The density of states just above $E_{\mathrm{F}}$ decreases with increasing amounts of $\mathrm{Sb}$ (Fig. 3b), as expected both from theory [4,5] and comparison with the $\mathrm{Sb} 3 \mathrm{~d}_{5 / 2}$ XAS spectra (Fig. 3a). As seen in Fig. 3d, the polarization above background spectra (with increasing $\mathrm{Sb}$ overlayer thickness) depends on the energy relative to $E_{\mathrm{F}}$, thus cannot be simply an attenuation of the clean $\mathrm{NiMnSb}(100)$. Further, the $\mathrm{Sb}$ atoms in the NiMnSb substrate lattice cannot be contributing to this polarization, as the MCD signal from the $\mathrm{Sb} 3 \mathrm{~d}_{5 / 2}$ core is negligible (Fig. 3c).

In Fig. 4, we have plotted the polarization asymmetry at $E_{\mathrm{F}}(\bigcirc)$, the average polarization from $E_{\mathrm{F}}$ to $0.5 \mathrm{eV}$ above $E_{\mathrm{F}}(\boldsymbol{\square})$ and the polarization integrated from $E_{\mathrm{F}}$ to $1.0 \mathrm{eV}$ above $E_{\mathrm{F}}(\Delta)$ with increasing $\mathrm{Sb}$ overlayer thickness. While the average polarization from $E_{\mathrm{F}}$ to $0.5 \mathrm{eV}$ above $E_{\mathrm{F}}(\boldsymbol{\square})$ and the polarization integrated from $E_{\mathrm{F}}$ to $1.0 \mathrm{eV}$ above $E_{\mathrm{F}}(\Delta)$, both show an exponential decline, as might be expected from a Landau-Ginzburg model, the measured polarization at $E_{\mathrm{F}}$ does not. For the thinnest 


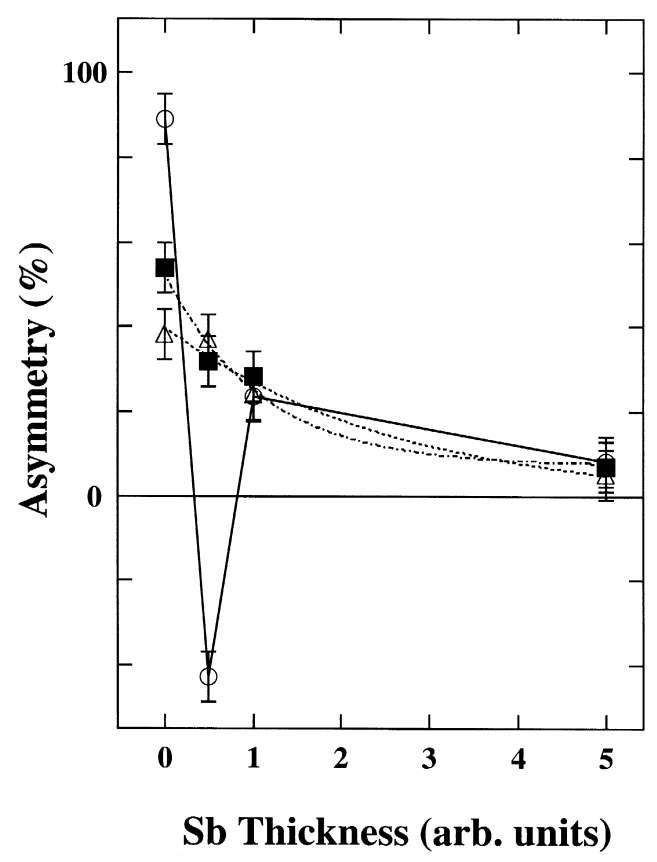

Fig. 4. The polarization asymmetry at $E_{\mathrm{F}}(\bigcirc)$, the average polarization from $E_{\mathrm{F}}$ to $0.5 \mathrm{eV}$ above $E_{\mathrm{F}}(\boldsymbol{\square})$ and the average polarization from $E_{\mathrm{F}}$ to $1.0 \mathrm{eV}$ above $E_{\mathrm{F}}(\Delta)$ have been plotted as a function of the relative $\mathrm{Sb}$ overlayer thickness. The figure is adapted from the data in Fig. 3. Only the average polarization from $E_{\mathrm{F}}$ to $0.5 \mathrm{eV}$ above $E_{\mathrm{F}}(\boldsymbol{\square})$ and the average polarization from $E_{\mathrm{F}}$ to $1.0 \mathrm{eV}$ above $E_{\mathrm{F}}(\Delta)$ show an exponential decline with increasing $\mathrm{Sb}$ overlayer thickness (dashed lines). Without an accurate estimate of the absolute values of the $\mathrm{Sb}$ overlayer thickness, no estimate can be made of the $\mathrm{Sb}$ paramagnetic correlation length from this data.

Sb overlayer studied, the polarization is negative. This suggests that there is an interface state between the $\mathrm{Sb}$ capping layer and $\mathrm{NiMnSb}(100)$ that is either antiferromagnetic (if thin enough), or antiferromagnetically aligned with the NiMnSb(100) substrate. Further, it is clear that some $\mathrm{Sb}$ unoccupied bands polarized more readily than others and the unoccupied states in the $\mathrm{Sb}$ overlayer are polarized differently than the $\mathrm{NiMnSb}(100)$ substrate at $\boldsymbol{k}_{\|}=0$.

The origin of the electronics structure, at the $\mathrm{Sb}-\mathrm{NiMnSb}(100)$ interface, cannot be determined from our data. Nonetheless, there are two possible contributions to the interface electronic structure should be considered: formation of MnSb-like electronic structure [35-38] at the interface and the possibility of a huge free energy difference at the interface. The latter contribution to interface states is suggested by measurements that demonstrate that the surface free energy is very different from the bulk [29].

While we cannot ascertain anything more than the relative thickness of our $\mathrm{Sb}$ overlayer (because of our sample preparation procedure), these spinpolarized inverse photoemission measurements show huge deviations from the behavior expected for simple polarization attenuation through a paramagnetic overlayer or from Landau-Ginzburg mean field models. Thus, while $\boldsymbol{k}_{\|}=0$ corresponds to long range magnetic order, polarization cannot be simply related to magnetization. As exchange splitting has now been shown to be wave vector, and band dependent, even for local moment systems [25,39], this is not entirely unexpected. What must be asked, in the future, is whether spin-polarized attenuation experiments through a paramagnetic overlayer, from a ferromagnetic substrate, can actually be used to determine the spin correlation length or spin dependent mean free path of the paramagnet, independent of wave vector or energy.

\section{Acknowledgements}

This work was supported by NSF through grant \# DMR-98-02126, the Center for Materials Research and Analysis (CMRA) and the Nebraska Research Initiative at the University of Nebraska, and the Region Rhone-Alpes through the 'Nanotechnologie' program under contract \#PR97024. The authors would like to thank Mircea Chipara for his assistance with the MOKE measurements.

\section{References}

[1] P.A. Dowben, D. LaGraffe, D. Li, A. Miller, L. Zhang, L. Dottl, M. Onellion, Phys. Rev. B 43 (1991) 3171.

[2] J. Mathon, J. Phys. F: Met. Phys. 16 (1986) L217.

[3] K. Binder, P.C. Hohenberg, Phys. Rev. B 6 (1972) 3461.

[4] X. Gonze, R. Sporken, J.P. Vigneron, R. Caudano, J. Ghijsen, R.L. Johnson, L. Ley, H.W. Richter, Phys. Rev. B 44 (1991) 11023.

[5] Yi Liu, R.E. Allen, Phys. Rev. B 52 (1995) 1566.

[6] R.A. de Groot, F.M. Mueller, P.G. van Engen, K.H.J. Buschow, Phys. Rev. Lett. 50 (1983) 2024.

[7] J.-S. Kang, J.H. Hong, S.W. Jung, Y.P. Lee, J.-G. Park, C.G. Olson, S.J. Youn, B.I. Min, Soldi State Commun. 88 (1993) 653. 
[8] I. Galanakis, S. Ostanin, M. Alouani, H. Dreysse, J.M. Wills, Phys. Rev. B 61 (2000) 4093.

[9] D. Ristoiu, J.P. Nozières, C.N. Borca, Takashi Komesu, Hae-kyung Jeong, P.A. Dowben, Europhys. Lett. 49 (2000) 624.

[10] R.J. Soulen, J.M. Byers, M.S. Osofsky, B. Nadgorny, T. Ambrose, S.F. Cheng, P.R. Broussard, C.T. Tanaka, J. Nowak, J.S. Moodera, A. Barry, J.M.D. Coey, Science 282 (1998) 85.

[11] G.L. Bona, F. Meier, M. Taborelli, E. Bucher, P.H. Schmidt, Solid State Commun. 56 (1985) 391

[12] C.T. Tanaka, J. Nowak, J.S. Moodera, J. Appl. Phys. 86 (1999) 6239.

[13] J.A. Caballera et al., J. Magn. Magn. Mater. 198-199 (1999) 55.

[14] K.E.H.M. Hanssen et al., IEEE Magnetics, 1997.

[15] C. Tanaka, J. Nowak, J.S. Moodera, J. Appl. Phys. 81 (1997) 5515.

[16] Ch. Horequin, E. Lelièvre-Berna, J. Pierre, Physica B 234236 (1997) 602.

[17] Jaewu Choi, Jiandi Zhang, S.-H. Liou, P.A. Dowben, E.W. Plummer, Phys. Rev. B 59 (1999) 13453.

[18] Jaewu Choi, C. Waldfried, S.-H. Liou, P.A. Dowben, J. Vac. Sci. Technol. A 16 (1998) 2950.

[19] Hani Dulli, P.A. Dowben, Jaewu Choi, S.-H. Liou, E.W. Plummer, Appl. Phys. Lett. 77 (2000), in press.

[20] C.N. Borca, Delia Ristoiu, Q.L. Xu, S.-H. Liou, S. Adenwalla, P.A. Dowben, Journ. Appl. Phys. 87 (2000) 6104.

[21] C.N. Borca, R.H. Cheng, Shane Stadler, Y.U. Idzerda, Hani Dulli, Jaewu Choi, D, N, Mcilroy, Q.L. Xu, S. H, Liou, Z.C. Zhong, P.A. Dowben, MRS Symposium Proc. 602 (2000), in press.

[22] K.P. Kämper, W. Schmitt, G. Güntherodt, R.J. Gambino, R. Ruf, Phys. Rev. Lett. 59 (1987) 2788.

[23] D. Ristoiu, J.P. Nozières, L. Ranno, J. Magn. Magn. Mater., 2000 , in press.
[24] F. Ciccaci, H.-J. Drouhin, C. Hermann, R. Houdré, G. Lampel, Appl. Phys. Lett. 54 (1989) 632.

[25] C. Waldfried, T. McAvoy, D. Welipitiya, Takashi Komesu, P.A. Dowben, E. Vescovo, Phys. Rev. B 58 (1998) 7434.

[26] Takashi Komesu, C. Waldfried, Hae-Kjung Jeong, D.P. Pappas, T. Rammer, M.E. Johnston, T.J. Gay, P.A. Dowben, in: G.T. Burnham, Xiaoguang He, Kurt J. Linden, S.C. Wang (Eds.), Laser Diodes and LEDs in Industrial, Measurement, Imaging and Sensor Applications II: Testing, Packaging, and Reliability of Semiconductor Lasers V, Proc. SPIE 3945 (2000) 6.

[27] J.H. Scofield, J. Elec. Spec. Rel. Phenom. 8 (1976) 129.

[28] M.P. Seah, M.E. Jones, M.T. Anthony, Surface and Interface Analysis 6 (1984) 242; M.P. Seah, Surface and Interface Analysis 20 (1993) 243.

[29] D. Ristoiu, J. P Nozières, C.N. Borca, B. Borca, P.A. Dowben, Appl. Phys. Lett. 76 (2000) 2349.

[30] C.N. Borca, R.H. Cheng, Q.L. Xu, S.H. Liou, S. Stadler, Y.U. Idzerda, P.A. Dowben, J. Appl. Phys. 87 (2000) 5606.

[31] H. Bethe, Ann. Phys. 5 (1930) 325.

[32] S. Tamura, C.J. Powell, D.R. Penn, Surf. Interface Anal. 17 (1991) 911

[33] S. Tamura, C.J. Powell, D.R. Penn, Surf. Interface Anal. 21 (1994) 165.

[34] C.J. Powell, A. Jablonski, J. Vac. Sci. Technol. A 17 (1999) 1122.

[35] R. Coehoorn, C. Haas, R.A. de Groot, Phys. Rev. B 31 (1985) 1980.

[36] H. Okuda, S. Senba, H. Sato, K. Shimada, H. Namatame, M. Taniguchi, J. Electron Spectrosc. Rel. Phenom. 101-103 (1999) 657.

[37] P. Ravidran, A. Delin, P. James, B. Johansson, J.M. Wills, R. Ahuja, O. Eriksson, Phys. Rev. B 59 (1999) 15680.

[38] O. Rader et al., Phys. Rev. B 57 (1998) R689.

[39] Takashi Komesu, C. Waldfried, P.A. Dowben, Phys. Lett. A 256 (1999) 81 